\title{
Cost-Effective Constructions for Nonblocking WDM Multicast Switching Networks
}

\author{
Dazhen Pan \\ Computer Science and Engineering Dept. \\ State University of New York at Buffalo \\ 201 Bell Hall, Amherst, NY 14260, USA \\ Email: dpan@cse.buffalo.edu
}

\author{
Vishal Anand \\ Computer Science Dept. \\ State University of New York \\ College at Brockport \\ 211A Faculty Office Building \\ Brockport NY 14420, USA \\ Email: vanand@brockport.edu
}

\author{
Hung Q. Ngo \\ Computer Science and Engineering Dept. \\ State University of New York at Buffalo \\ 201 Bell Hall, Amherst, NY 14260, USA \\ Email: hungngo@cse.buffalo.edu
}

\begin{abstract}
Constructing wavelength division multiplexing (WDM) multicast switches with cheap components and low complexity is an important problem in optical networking, since multicasting is one of the key requirements of emerging bandwidth-intensive applications such as video conferencing, video on demand and online gaming.

In this paper we present several new cost-effective designs for constructing non-blocking WDM multicast switching networks. More specifically, we provide a wide-sense nonblocking switch architecture, which uses fewer semiconductor optical amplifiers than existing architectures. In addition, for the first time we have presented a rearrangeably nonblocking WDM switch architecture using array waveguide grating routers and wavelength converters.
\end{abstract}

\section{INTRODUCTION}

Despite the recent downturn in the telecommunication industry in general and the optical networking sector in particular, Internet traffic is still doubling every year. In order to meet the ever increasing bandwidth demand from a large number of users in scientific computing and academic communities, as well as in military and other government agencies, there is a renewed interest in photonic switching as evident from several ongoing and planned national-scale projects in the US, Europe, and Asia (see for example, DARPA's recent BAA on Data in the Optical Domain Networks http://www.darpa.mil/baa/BAA03-19.htm).

Optical networks using the wavelength-division multiplexing (WDM) technology [1] enable a single strand of fiber to have an aggregate bandwidth of terabits per second [2][4]. Further, all-optical WDM networks wherein the user data travels entirely in the optical domain eliminate expensive optical-electrical-optical (OEO) conversions, allow unprecedented transmission rates. Thus bandwidth-intensive applications such as HDTV, video-on-demand services, distributed on-line gaming etc. are now possible.

Multicasting is the ability to transmit information from a single source node to multiple destination nodes. The aforementioned bandwidth-intensive applications require multicast services to efficiently use the network resources, such as fiber bandwidth and switches at the nodes.
In this paper, we give several new cost-effective nonblocking WDM switch designs for multicasting. Both rearrangeably nonblocking and widesense nonblocking designs are to be presented. To the best of our knowledge, this is the first paper to address rearrangeably nonblocking WDM multicast switching networks. As pointed out in [?], [5], rearrangeably nonblocking switches are very useful for optical packet/burst switching networks where optical packets/bursts are switched synchronously or one batch at a time.

Known works [6], [7] on WDM multicast switching networks have used ideas from multicast switching constructions in circuit switching networks [8]. Our constructions in this paper follow this line of research from a different angle, applying ideas from multicast circuit switching network constructions in [8]-[10]. We also use several new ideas proposed in [?] for constructing unicast WDM switches.

The rest of the paper is organized as follows. Section II gives basic concepts and definitions. Sections III and IV present our rearrangeably and widesense nonblocking nonblocking constructions, respectively. The last section compares our designs with known designs, and concludes the paper.

\section{PRELIMINARY}

\section{A. Basic concepts}

Let's consider a WDM cross-connect (WXC) with $f$ input fibers and $f$ output fibers. Each fiber is capable of carrying a set of $w$ wavelengths. Let $I_{x y}$ denote the $y^{t h}$ wavelength on input fiber $x$, and $O_{x y}$ the $y^{t h}$ wavelength on output fiber $x$, where $1 \leq x \leq f$, and $1 \leq y \leq w$.

In multicast traffic, it is often not necessary to have the same multicast connection coming out on the same output port on different wavelengths [6], [11]. Consequently, we consider the request model in which each multicast request is from an input wavelength to a set of wavelengths on different output fibers.

A multicast connection request is of the form $\left(I_{x y}, \mathcal{A}\right)$, where $\mathcal{A}=\left\{O_{x_{1} y_{1}}, \cdots, O_{x_{k} y_{k}}\right\}$ denote a set of $k$ output wavelengths, where $x_{i} \neq x_{j}$ for $i \neq j$. The multicast request indicates that a connection (with fanout $k$ ) is to be established from input wavelength $I_{x y}$ to the set of $k$ output wavelengths in $\mathcal{A}$. 
Consider a WXC with a few multicast connection requests already established. A new request $\left(I_{x y}, \mathcal{A}\right)$ is said to be valid if the input wavelength $I_{x y}$ is free and all the requested wavelengths in $\mathcal{A}$ are also free. A request frame is a set of requests such that no two requests are from the same wavelength in the same input fiber, and there are at most $w$ requests to any output fiber. A request frame is realizable by a WXC if all requests in the frame can be routed simultaneously. A WXC is rearrangeably nonblocking iff any request frame is realizable. A WXC is wide-sense nonblocking iff a new valid request can always be routed through the WXC without disturbing existing connections as long as the routes are picked by a specific routing algorithm.

\section{B. WDM Crossbar with multicast capability}

To perform multicasting in a WDM switching network, we need switching components which are capable of doing multicasting. The WDM multicast crossbar proposed in [6] is one such component, which made use of splitters, SOAs and combiners. We will use MCB to denote multicast crossbar. Figure 1 shows a possible construction of an $N \times M$ MCBs. The input and output fibers are meant to carry only one wavelength on each.

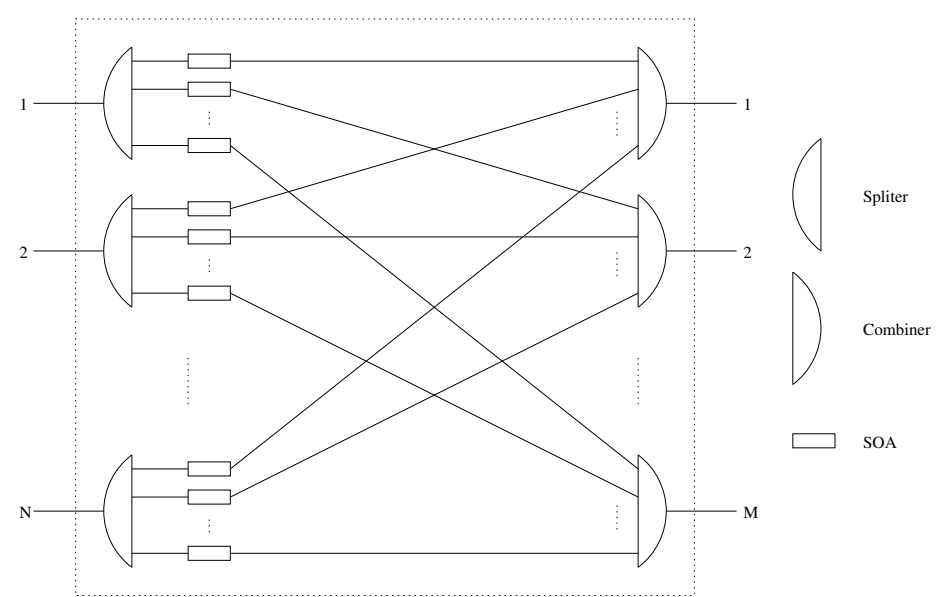

Fig. 1. A possible implementation of an $N \times M$ multicast crossbar (MCB).

\section{Arrayed Waveguide Grating Routers}

Passive arrayed waveguide grating routers (AWGRs) are attractive optical switching components because they are commercially available and inexpensive, relatively simple to fabricate, and they do not consume any power [12], [13].

An $M \times M$ AWGR on $w$ wavelengths has a fixed routing pattern defined as follows. Number the inputs and outputs from 0 to $M-1$, and the wavelengths from 0 to $w-1$. Then, an input signal on wavelength $i$ at input fiber $j$ gets routed to the same wavelength on output fiber $((i-j) \bmod M)$.

Remark II.1. Some authors define the output line to be $((j-i) \bmod M)$ instead. This is not a discrepancy, as we can always re-number the wavelengths and input lines. For example, renaming $\lambda_{i}$ by $\lambda_{M-1-i^{\prime}}$, and input $j$ by $\left(M-1-j^{\prime}\right)$, we have $(i-j)=\left(j^{\prime}-i^{\prime}\right)$.
An AWGR can be used to construct a unicast WXC (without multicasting capability) by simply placing a wavelength converter (WC) at each input.

\section{REARRANGEABLY NONBLOCKING CONSTRUCTION}

Using a single MCB to construct a multicast switching network requires too many switching components ( $f^{2} w^{2}$ SOAs). To reduce the cost of the network, a multistage design, using the idea of 3-stage Clos network [14] can be adopted, as was done in [6]. However, increasing the number of stages also increases the delay and attenuation incurred by the signals. Hence, a design with few stages and low cost is preferred. In this section and the next, we present our nonblocking multicast switching architectures which have only 2 stages and a cost comparable with existing constructions.

\section{A. RNB construction with first stage having multicast switch-} ing capability

We use WXC-RNB-1 to denote the construction given in this subsection. A general construction of WXC-RNB1 is shown in figure 2. The black solid squares represent full-range wavelength converters. There are no multiplexers/demultiplexers in the first two stages, except at the input and output fibers. The MCBs can be set up so that each link carries at most one wavelength. We can now treat this network as a traditional circuit switching network, similar to the 3-stage Clos network. Our other designs are also based on this idea, which was first observed in [?].

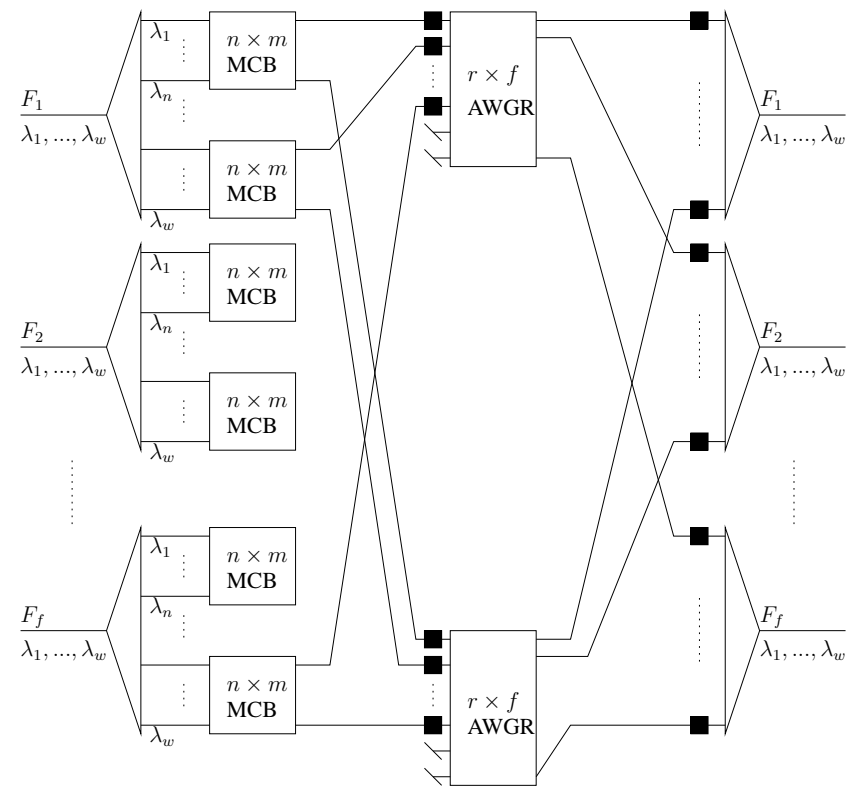

Fig. 2. A general construction for WXC-RNB-1, since $r \neq f$, some inputs or outputs of AWGR may be unused. The solid black squares represent full wavelength converters.

Remark III.1. Note that our designs do not have a third stage as in the regular Clos networks. This is because under our request model, after the second stage the request is already routed 
to the requested fiber. Consequently, no further switching capability is required after the second stage. Again, all of our designs follow this idea.

Theorem III.2. WXC-RNB-1 is rearrangeably nonblocking if $m \geq \max \{n f, w\}$

Proof. Since we only allow one wavelength on each link, this construction will be equivalent to its circuit switching counterpart. The results of nonblocking condition of a traditional multicast switching network can be directly applied here. A 3 -stage Clos network $C\left(n_{1}, r_{1}, m, n_{2}, r_{2}\right)$, with 1 st and 3 rd stages having multicast switching capability, is rearrangeably nonblocking if $m \geq \max \left\{\min \left\{n_{1} r_{2}, n_{1} r_{2}\right\}, \min \left\{n_{2}, n_{1} r_{1}\right\}\right\}$ [9], [15]. Since WXC-RNB-1 does not have a true 3rd stage, we ignore this stage. The AWGRs with WCs in the 2nd stage are equivalent to RNB crossbars. When a connection reaches the destination fiber through some link, the WC on that link can be used to convert this wavelength to the requested wavelength on that fiber. With respect to our construction, we have $n_{1}=n, r_{1}=r, n_{2}=w, r_{2}=f$. Substituting these values in the above equation, we get $m \geq \max \{n f, w\}$.

By varying the value of $n$ we can optimize the cost of the switch. The difference between $w$ and $f$ could vary depending on the environment employing a WXC. For example, in metropolitan area networks (MANs), coarse wavelength division multiplexing (CWDM) with few wavelengths per fiber is cost efficient, hence we may have $f \geq w$. On the other hand, in wide area networks (WANs), to achieve cost efficiency carriers typically employ WDM or dense WDM, and hence we have $f \leq w$. We analyze the cost of our constructions for both the cases. The cost of the switch is dominated by the number of SOAs and WCs, since they are much more expensive to manufacture than splitters, combiners, and AWGR. Moreover, SOAs are power-consuming and difficult to fabricate in large numbers.

In the following we use \#SOA to denote the number of $S O A$ s and $\# W C$ for $W C$ s.

Corollary III.3. For WXC-RNB-1, we have:

$$
\begin{aligned}
& \# S O A \leq\left\{\begin{array}{l}
f^{2} w=O\left(f^{2} w\right) \quad \text { if } f \geq w, \\
f w^{2}=O\left(f w^{2}\right) \quad \text { if } f<w
\end{array}\right. \\
& \# W C \leq\left\{\begin{array}{l}
f^{2} w+f^{2}=O\left(f^{2} w\right) \quad \text { if } f \geq w, \\
2 f^{2} w+f w=O\left(f^{2} w\right) \quad \text { if } f<w
\end{array}\right.
\end{aligned}
$$

Proof. In this construction, we have

$$
\begin{aligned}
\# S O A & =r n m \\
\# W C & =r m+m f
\end{aligned}
$$

Hence, we can let $n=1, r=f w$, if $f \geq w$.

For the case where $f<w$, let us assume that $f$ divides $w$ for the sake of clarity. Normally this is true in practice, since $f, w$ are often powers of 2 . Even if $f$ does not divide $w$, the results would only be increased by a constant factor. Letting $n=\frac{w}{f}, r=\frac{f w}{n}=f^{2}$, then $m=w, \# S O A=r n m=$ $f w^{2}$.

B. RNB construction with 2 nd stage having multicast switching capability

The construction in this subsection, denoted by WXC-RNB2 , is very similar to WXC-RNB-1, except that now we have MCBs in the 2nd stage and AWGRs in 1st stage, the remaining connecting pattern will be the same. Note that now the 2 nd stage has multicast switching capability while the 1st does not.

Theorem III.4. $W X C-R N B-2$ is rearrangeably nonblocking if $m \geq n+\sqrt{f w(w-1)}$.

Proof. The proof is similar to Theorem III.2. A 3-stage Clos network $C\left(n_{1}, r_{1}, m, n_{2}, r_{2}\right)$, with 2 nd and 3 rd stages having multicast switching capability, is rearrangeably nonblocking if $m \geq n_{1}+\sqrt{n_{2} r_{2}\left(n_{2}-1\right)}$ [9]. Substituting for $n, f, w$, we get the theorem.

Corollary III.5. For WXC-RNB-2, we have:

$$
\begin{aligned}
& \# S O A \leq 2 f^{2} w=O\left(f^{2} w\right) \\
& \# W C \leq f w+2 f \sqrt{f w^{2}-f w} \leq f w+2 f^{\frac{3}{2}} w=O\left(f^{\frac{3}{2}} w\right)
\end{aligned}
$$

Proof. In this construction, we have

$$
\begin{aligned}
\# S O A & =m r f \\
\# W C & =f w+m f
\end{aligned}
$$

To minimize $m$, let $n=\sqrt{f w^{2}-f w}$.

\section{WIDE-SENSE NONBLOCKING CONSTRUCTION}

In this section we present 2 new WSNB constructions. One is based on our 2-stage construction (denoted as WXCWSNB-1), while the other will is a 3-stage design, which is an extension of the constructions in [6] (denoted as WXCWSNB-2).

\section{A. Two-stage WSNB construction}

A general construction of WXC-WSNB-1 is shown in figure 3. Again, each link (except the input and output fibers) carries at most one wavelength.

Theorem IV.1. WXC-WSNB-1 is wide-sense nonblocking if

$$
\begin{array}{ll}
m>2(n-1) \frac{\lg f}{\lg \lg f}+(w-1) \sqrt{(\lg f)} & \text { when } f \geq w \\
m>(n-1) \lg f+2(w-1) & \text { when } f<w(
\end{array}
$$

Proof. As noted earlier, in our construction we can remove the third stage switches, and this construction remains equivalent to a regular 3-stage Clos network. Hence, existing results on multicast 3-stage Clos network can be applied.

Yang [8] showed that a 3-stage Clos network $C\left(n_{1}, r_{1}, m, n_{2}, r_{2}\right)$, is wide-sense nonblocking if

$$
m>2\left(n_{1}-1\right) \frac{\lg r_{2}}{\lg \lg r_{2}}+\left(n_{2}-1\right) \sqrt{\lg r_{2}}
$$




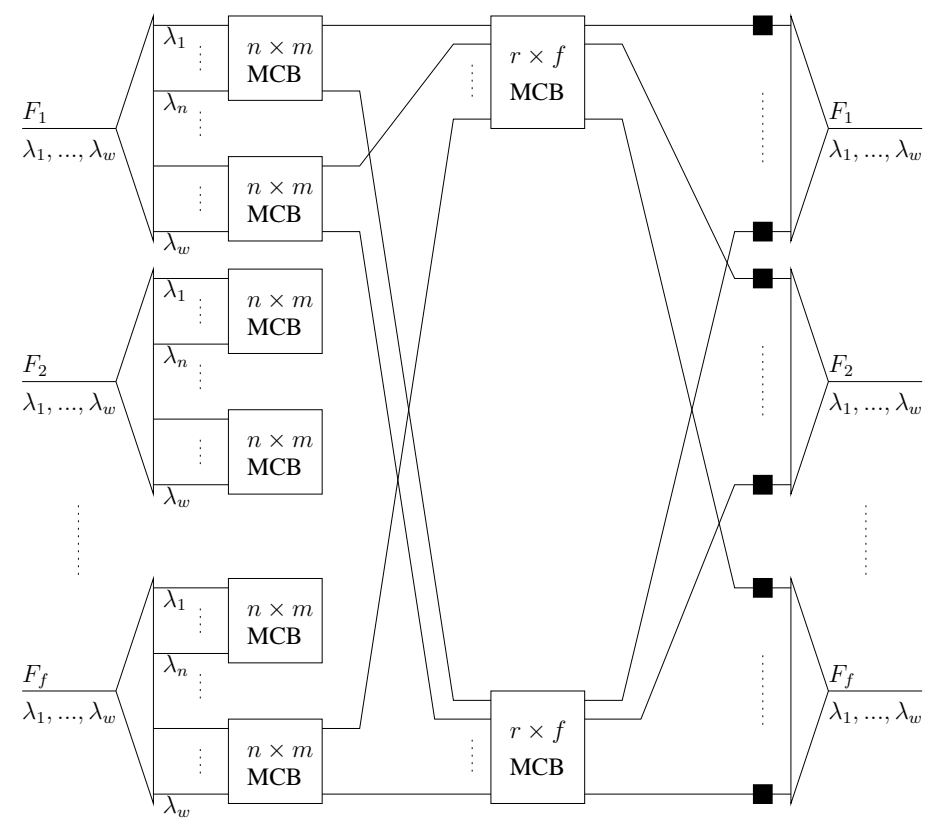

Fig. 3. A general construction for WXC-WSNB-1. The solid black squares represent full wavelength converters.

A similar bound is shown in [9]:

$$
m>\left(n_{1}-1\right)\left\lceil\lg r_{2}\right\rceil+2\left(n_{2}-1\right)
$$

which guarantees the network is WSNB when $\lg r_{2} \leq 2 n_{2}-1$. Combining these two results and substituting $n_{1}=n, r_{1}=$ $r, n_{2}=w, r_{2}=f$, we get the theorem.

Corollary IV.2. To make WXC-WSNB-1 wide-sense nonblocking, the following numbers of SOAs and WCs are sufficient

$$
\begin{aligned}
& \# S O A= \begin{cases}O\left(f^{2} w \frac{\lg f}{\lg \lg f}\right) & \text { if } f \geq w \\
O(f w \max (f \lg f, w)) & \text { if } f<w\end{cases} \\
& \# W C= \begin{cases}O\left(f^{2} \frac{\lg f}{\lg \lg f}\right) & \text { if } f \geq w \\
O(f \max (f \lg f, w)) & \text { if } f<w\end{cases}
\end{aligned}
$$

Proof. These bounds can be achieved by letting $n=f, r=w$. For $f<w$, we need

$$
\begin{aligned}
m & =2(f-1) \frac{\lg f}{\lg \lg f}+(w-1) \sqrt{(\lg f)}+1 \\
& =O\left(f \frac{\lg f}{\lg \lg f}\right) \\
\# S O A & =r n m+m r f=2 f w m=O\left(f^{2} w \frac{\lg f}{\lg \lg f}\right) \\
\# W C & =m f=f^{2} \frac{\lg f}{\lg \lg f}
\end{aligned}
$$

For $f<w$, we need

$$
\begin{aligned}
m & =(f-1) \lg f+2 w-1=O(\max (f \lg f, w)) \\
\# S O A & =r n m+m r f=2 f w m=O\left(\max \left(f^{2} w \frac{\lg f}{\lg \lg f}, f w^{2}\right)\right) \\
\# W C & =m f=O\left(\max \left(f^{2} \lg f, f w\right)\right)
\end{aligned}
$$

\section{B. Three-stage WSNB construction}

Yang et al. [6] proposed another request model in which a multicast connection request with source wavelength $\lambda$ can only request the same destination wavelength $\lambda$ on the output fibers. In terms of our request format $\left(I_{x y}, \mathcal{A}\right)$, this means that $\mathcal{A}$ will have the form $\left\{O_{x_{1} y}, \cdots, O_{x_{k} y}\right\}$, i.e. $\lambda_{\text {in }}=\lambda_{\text {out }}$.

With similar ideas as the ones in the previous subsection and the ideas in [6], we can give a WSNB construction for the multicast with same wavelength model (see [6]) using only $O\left(f^{\frac{3}{2}} w \frac{\lg f}{\lg \lg f}\right) S O A$ s and no WCs. In this construction, which we refer to as WXC-WSNB-2, there is a reserved link between the third stage and the multiplexer for each wavelength. By using a full wavelength converter on each of these links before they are multiplexed into the output fiber, we can use this construction to satisfy a request in our original request model, as we can always route a request originating at wavelength $\lambda$ by routing it to the link reserved for $\lambda$ on the requested fibers and then convert it to the requested wavelength.

Compared with the construction of [6] under our original request model (i.e., where $\lambda_{i n}$ need not necessarily be equal to $\lambda_{\text {out }}$ ), WXC-WSNB-2 uses tunable-input-tunable-output $W C$ s instead of tunable-input-fixed-output $W C$ s to save more $S O A$ s in the third stage. However, a tunable-input-tunable-output $W C$ can always be implemented by combining a tunable-inputfixed-output $W C$ and a fixed-input-tunable-output $W C$, thus reducing the cost.

\section{CONCLUSION AND FUTURE WORK}

In this paper we consider the problem of designing costefficient multicast capable switches for WDM optical networks. The work in [6] proposed a wide-sense nonblocking architecture which required $O\left(f^{\frac{3}{2}} w^{\frac{3}{2}} \frac{\lg f}{\lg \lg f}\right)$ SOAs when $f \geq$ $w$; this bound is achieved by taking $n=\sqrt{\frac{f}{w+1}}$ as in [16]. When $f<w$ the above condition cannot be satisfied, and $O\left(f^{\frac{3}{2}} w^{2} \frac{\lg f}{\lg \lg f}\right)$ SOAs are required. The number of WCs used is $O(f w)$ in both cases.

As compared to the above constructions, our 2-stage construction uses only $O(f w \max (f \lg f, w))$ SOAs when $f<w$ and the same number of WCs when $w \geq \lg f$. Further, note that our 2-stage designs use multiplexers and demultiplexers only for the input and output fibers, which is much less than the design in [6] which uses multiplexers and demultiplexers at each of its 3 stages. We have, also, for the first time, proposed RNB multicast WXC architectures for WDM networks. Tables I and II compare the cost of the proposed WXC in terms of the number of stages, SOAs, WCs and AWGRs.

\section{ACKNOWLEDGEMENT}

Hung Q. Ngo is partially supported by NSF CAREER Award CCF-0347565.

\section{REFERENCES}

[1] B. Mukherjee, Optical Communication Networks. New York, NY: McGraw-Hill, 1997. 


\begin{tabular}{|c||c|c|c|c|}
\hline & \#Stages & \#SOAs & \#WCs & \#AWGRs \\
\hline \hline WXC-RNB-1 & 2 & $O\left(f^{2} w\right)$ & $O\left(f^{2} w\right)$ & $f(f w) \otimes f$ \\
\hline WXC-RNB-2 & 2 & $O\left(f^{2} w\right)$ & $O\left(f^{\frac{3}{2}} w\right)$ & $O(\sqrt{f})(\sqrt{f} w) \otimes(2 \sqrt{f} w)$ \\
\hline \hline WXC-WSNB-1 & 2 & $O\left(f^{2} w \frac{\lg f}{\lg \lg f}\right)$ & $O\left(f^{2} \frac{\lg f}{\lg \lg f}\right)$ & N/A \\
\hline WXC-WSNB-2 & 3 & $O\left(f^{\frac{3}{2}} w \frac{\lg f}{\lg \lg f}\right)$ & $O(f w)$ & N/A \\
\hline Ref [6] & 3 & $O\left(f^{\frac{3}{2}} w^{\frac{3}{2}} \frac{\lg f}{\lg \lg f}\right)$ & $O(f w)$ & N/A \\
\hline
\end{tabular}

TABLE I

THE COST COMPARISON BETWEEN DIFFERENT CONSTRUCTIONS FOR $f \geq w$

\begin{tabular}{|c||c|c|c|c|}
\hline & \#Stages & \#SOAs & \#WCs & \#AWGRs \\
\hline \hline WXC-RNB-1 & 2 & $O\left(f w^{2}\right)$ & $O\left(f^{2} w\right)$ & $f(f w) \otimes f$ \\
\hline WXC-RNB-2 & 2 & $O\left(f^{2} w\right)$ & $O\left(f^{\frac{3}{2}} w\right)$ & $O(\sqrt{f})(\sqrt{f} w) \otimes(2 \sqrt{f} w)$ \\
\hline \hline WXC-WSNB-1 & 2 & $O(f w \max (f \lg f, w))$ & $O(f \max (f \lg f, w))$ & N/A \\
\hline WXC-WSNB-2 & 3 & $O\left(f^{\frac{3}{2}} w \frac{\lg f}{\lg \lg f}\right)$ & $O(f w)$ & N/A \\
\hline Ref [6] & 3 & $O\left(f^{\frac{3}{2}} w^{2} \frac{\lg f}{\lg \lg f}\right)$ & $O(f w)$ & N/A \\
\hline
\end{tabular}

TABLE II

THE COST COMPARISON BETWEEN DIFFERENT CONSTRUCTIONS FOR $f<w$

[2] Lucent Technologies Press Release, "Lucent Technologies unveils untra-high-capacity optical system; Time Warner Telecom first to announce it will deploy the system," 2001. http://www.lucent.com/press/0101/010117.nsa.html.

[3] Lucent Technologies Press Release, "Lucent Technologies engineer and scientists set new fiber optic transmission record," 2002. http://www.lucent.com/press/0302/020322.bla.html.

[4] Lucent Technologies Website, "What is dense wave division multiplexing (DWDM)," $2002 . \quad$ http://www.belllabs.com/technology/lightwave/dwdm.html.

[5] C. Qiao and M. Yoo, "Optical burst switching (obs) - a new paradigm for an optical internet," Journal of High Speed Networks, vol. 8, no. 1, pp. 69-84, 1999.

[6] Y. Yang, J. Wang, and C. Qiao, "Nonblocking WDM multicast switching networks," IEEE Trans. Para. and Dist. Sys., vol. 11, pp. 1274-1287, Dec 2000.

[7] X. Qin and Y. Yang, "A cost-effective construction for WDM multicast switching networks," in Proceedings of the 2002 IEEE International Conference on Communications (ICC 2002), vol. 5, (New York), pp. 2902-2906, IEEE, 1979.

[8] Y. Yang and G. M. Masson, "Nonblocking broadcast switching networks," IEEE Trans. Comput., vol. 40, no. 9, pp. 1005-1015, 1981.

[9] D. G. Kirkpatrick, M. Klawe, and N. Pippenger, "Some graph-colouring theorems with applications to generalized connection networks," SIAM J. Algebraic Discrete Methods, vol. 6, no. 4, pp. 576-582, 1985.

[10] D. Nassimi and S. Sahni, "Parallel permutation and sorting algorithms and a new generalized connection network," J. Assoc. Comput. Mach., vol. 29, no. 3, pp. 642-667, 1982.

[11] Y. Wang and Y. Yang, "Multicasting in a class of multicast-capable WDM networks," J. Lightwave Technol., vol. 13, pp. 128-141, Feb 2002.

[12] C. Dragone, "An $n \times n$ optical multiplexor using a planar arrangment of two star couplers," IEEE Photonic Technology Letters, vol. 6, pp. 812815, 1991

[13] J. Ramamirtham and J. S. Turner, "Design of wavelength converting switches for optical burst switching," in Proceedings of the 21st Annual Joint Conference of the IEEE Computer and Communications Societies (INFOCOM), vol. 2, pp. 1162-1171, IEEE, 2002.

[14] C. Clos, "A study of non-blocking switching networks," Bell System Tech. J., vol. 32, pp. 406-424, 1953.

[15] F. K. Hwang, The mathematical theory of nonblocking switching networks. River Edge, NJ: World Scientific Publishing Co. Inc., 1998.

[16] X. Qin and Y. Yang, "Nonblocking WDM switching networks with full and limited wavelength conversion," in Proceedings of 10th IEEE International Conference on Computer Communications and Networks (IC3N '01, Phoenix, AZ), vol. 5, (New York), pp. 48-54, IEEE, Oct 2001. 